\title{
Chopped sample heating for quantitative profile analysis of low energy electron diffraction spots at high temperatures
}

P. Kury, P. Zahl, M. Horn-von Hoegen, C. Voges, H. Frischat, H.-L. Günter, H. Pfnür, and M. Henzler

Citation: Review of Scientific Instruments 75, 4911 (2004);

View online: https://doi.org/10.1063/1.1807003

View Table of Contents: http://aip.scitation.org/toc/rsi/75/11

Published by the American Institute of Physics

\section{Articles you may be interested in}

Third-generation conical spot profile analyzing low-energy electron diffraction

Review of Scientific Instruments 73, 2958 (2002); 10.1063/1.1489074

Determination and correction of distortions and systematic errors in low-energy electron diffraction

Review of Scientific Instruments 84, 015111 (2013); 10.1063/1.4774110

Lost in reciprocal space? Determination of the scattering condition in spot profile analysis low-energy electron diffraction

Review of Scientific Instruments 82, 035111 (2011); 10.1063/1.3554305

Reciprocal space mapping by spot profile analyzing low energy electron diffraction

Review of Scientific Instruments 76, 085102 (2005); 10.1063/1.1988287

Comparisons between PZT and PVDF thick films technologies in the design of low-cost pyroelectric sensors

Review of Scientific Instruments 75, 4906 (2004); 10.1063/1.1807002

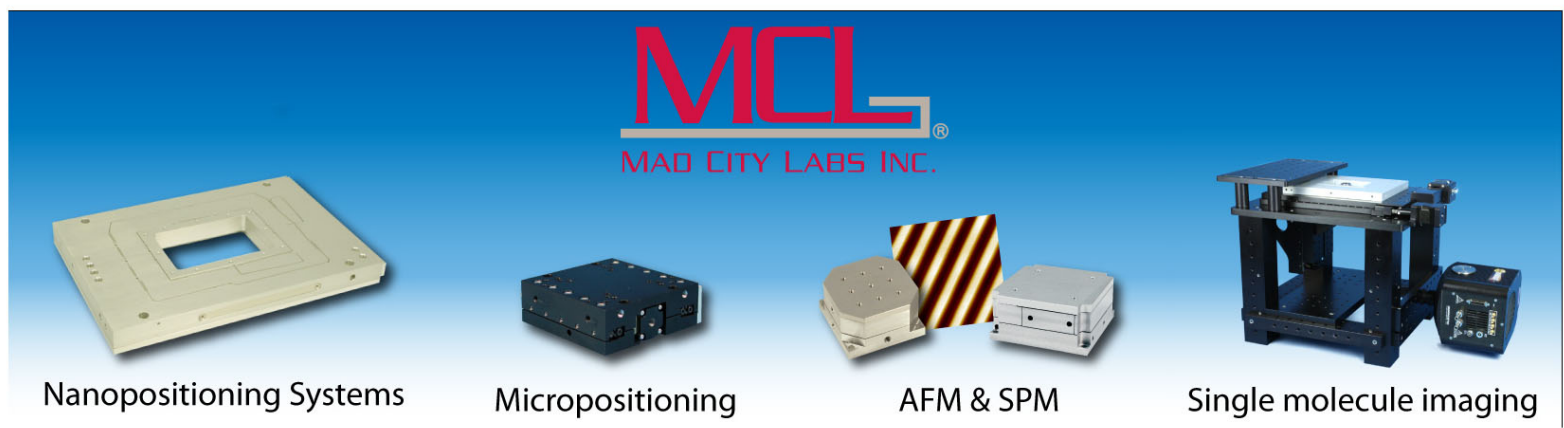




\title{
Chopped sample heating for quantitative profile analysis of low energy electron diffraction spots at high temperatures
}

\author{
P. Kury, ${ }^{\text {a) }}$ P. Zahl, ${ }^{\text {b) }}$ and M. Horn-von Hoegen \\ Institut für Laser- und Plasmaphysik, Universität Duisburg-Essen, D-45117 Essen, Germany \\ C. Voges, H. Frischat, H.-L. Günter, H. Pfnür, and M. Henzler \\ Institut für Festkörperphysik, Abt. Oberflächen, Universität Hannover, D-30167 Hannover, Germany
}

(Received 22 January 2004; accepted 6 July 2004; published 2 November 2004)

\begin{abstract}
Spot profile analysis low energy electron diffraction (SPA-LEED) is one of the most versatile and powerful methods for the determination of the structure and morphology of surfaces even at elevated temperatures. In setups where the sample is heated directly by an electric current, the resolution of the diffraction images at higher temperatures can be heavily degraded due to the inhomogeneous electric and magnetic fields around the sample. Here we present an easily applicable modification of the common data acquisition hardware of the SPA-LEED, which enables the system to work in a pulsed heating mode: Instead of heating the sample with a constant current, a square wave is used and electron counting is only performed when the current through the sample vanishes. Thus, undistorted diffration images can be acquired at high temperatures. (C) 2004 American Institute of Physics. [DOI: 10.1063/1.1807003]
\end{abstract}

\section{INTRODUCTION}

The discovery of the diffraction of electrons by Davisson and Germer ${ }^{1}$ paved the way for one of the most successful techniques for the investigation of surfaces: low energy electron diffraction (LEED). Its surface sensitivity is caused by the short mean free path of electrons in solids of only a few atomic monolayers, ${ }^{2}$ and its versatility arises from the easy tunability of the wavelength of the electrons via their energy. The LEED method became usable for reliable quantitative analysis of surface structures and morphology with the development of the spot profile analysis-LEED (SPA-LEED). ${ }^{3,4}$ While optical LEED instruments with spherical grids and screen $^{5-8}$ are geometrical representations in real space of the Ewald sphere construction in reciprocal space, the mode of operation of the SPA-LEED can be interpreted as scanning the reciprocal space via an electrostatic deflection system over a single electron detector (channeltron ${ }^{22}$ ). This results in a much higher dynamic range and in a higher resolution, so that the profiles of diffraction spots can be evaluated quantitatively in order to gain information about structure and morphology of surfaces. The capabilities of the SPA-LEED have been reviewed in detail by Horn-von Hoegen, ${ }^{9}$ and the latest upgrades of this instrument, such as the third generation conical shaped SPA-LEED system [which greatly improves the accessibility of the sample to molecular beam epitaxy (MBE) sources, see Fig. 1] and the real-space mode ${ }^{10}$ show the potential of the method for the future.

Unfortunately, the motion of electrons with low energies between some ten and a few hundred electron volts $(\mathrm{eV})$ is strongly affected by electric and magnetic fields. Therefore, several methods are used for the reduction of these fields: Only nonmagnetic materials should be used near the sample,

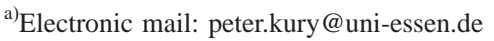

${ }^{b)}$ Present address: IBM Zurich Research Laboratory, Switzerland.
}

the chamber can be shielded against magnetic stray fields with a high permeability material ( $\mu$ metal), and in order to reduce the effects of electric fields across the sample, a potentiometer should be wired in parallel to the sample with its slider connected to ground potential, so that the ground potential point can be set to that position on the sample where the electron beam hits.

In many applications, especially those dealing with silicon samples at high temperatures, a direct current sample heating has significant advantages in contrast to electron bombardment, radiation heating or heating pads:

- The maximum temperature is as high as the melting point. Good sample cleaning is achieved, since no other material is in contact with the sample, and no hot, outgassing filament is next to it.

- No shielding against stray electrons is necessary.

- Only one low voltage power supply and only two electrical feedthroughs into the vacuum are needed.

A big disadvantage, however, is that a directly heated sample is surrounded by electrical and magnetic fields that disturb the LEED measurements: In setups with complicated sample geometries (like that for surface stress measurements ${ }^{11,12}$ ) the fields around the sample can be especially extremely inhomogeneous, and if metal samples have to be heated, large currents of some ten amperes with high corresponding magnetic fields are used.

Thus, a method is desirable which reveals the full potential of the SPA-LEED even at high temperatures while keeping the above-mentioned advantages of direct current sample heating. We label this method the "chopped sample heating" method: If the sample is heated with square wave pulses instead of a constant direct current (dc), then it is free of the disturbing fields whenever the current and voltage on the sample vanish. With a modification of the SPA-LEED data 


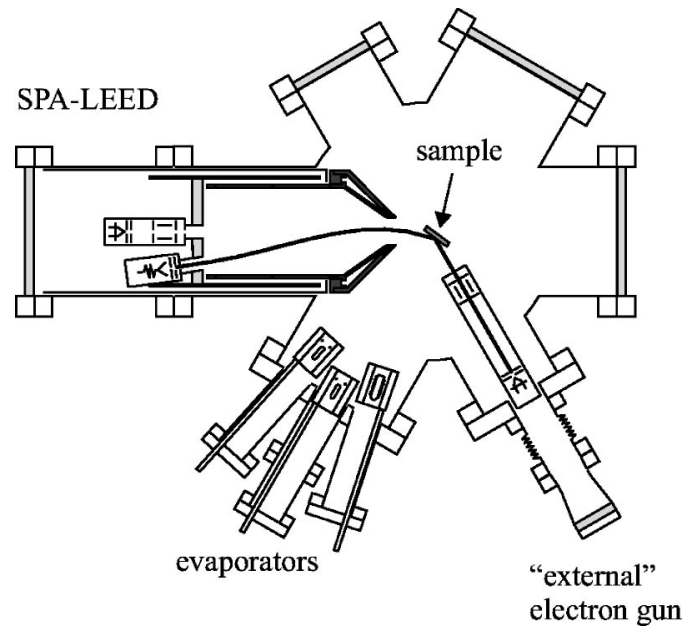

FIG. 1. Cross section through an ultra high vacuum (UHV) chamber, showing a typical SPA-LEED setup with the so-called "external" electron gun [which allows the in situ monitoring of thin film growth processes as with reflection high energy electron diffraction (RHEED)], and the path of the electrons between the filament and the channeltron.

acquisition hardware, it is possible to record only those electrons that have been reflected off the sample in such a fieldfree period.

In principle, this technique is not restricted to square wave pulses; decaying sine waves (also known as degauss signals) may be better suited for applications with ferromagnetic materials, and a high frequency alternating current (ac) can be useful if effects like electromigration should be avoided. However, because of their much more complicated switching behavior, the use of those signals for the heating will not be treated here.

\section{REALIZATION OF THE CHOPPED HEATING}

There are two alternatives that allow the detection of electrons that have been scattered from the field-free sample in a period between two heating pulses: using either a pulsed electron source, i.e., an electron gun with a fast beam blanker, or pulsed electron detection. To avoid modifications of components inside the ultra high vacuum (UHV), the second alternative as sketched in Fig. 2 was chosen. The necessary minor and reversible changes of the commonly used data acquisition hardware will be described below.

\section{A. Cooling speed of the sample}

The time constant of the chopper must be chosen in accordance with the maximum cooling speed of the sample in order to ensure a constant and homogeneous temperature distribution.

If the sample's temperature distribution is homogenous then the thermal conduction through the sample holder is small and can be neglected. Because the sample is placed inside a UHV chamber, convection is also neglegible and the loss of heat energy by radiation with its $T^{4}$ dependence is not only the fastest but also the dominating process. Therefore it is sufficient for the estimation of the cooling speed. In a high-pressure chemical vapor phase deposition (CVD) envi-

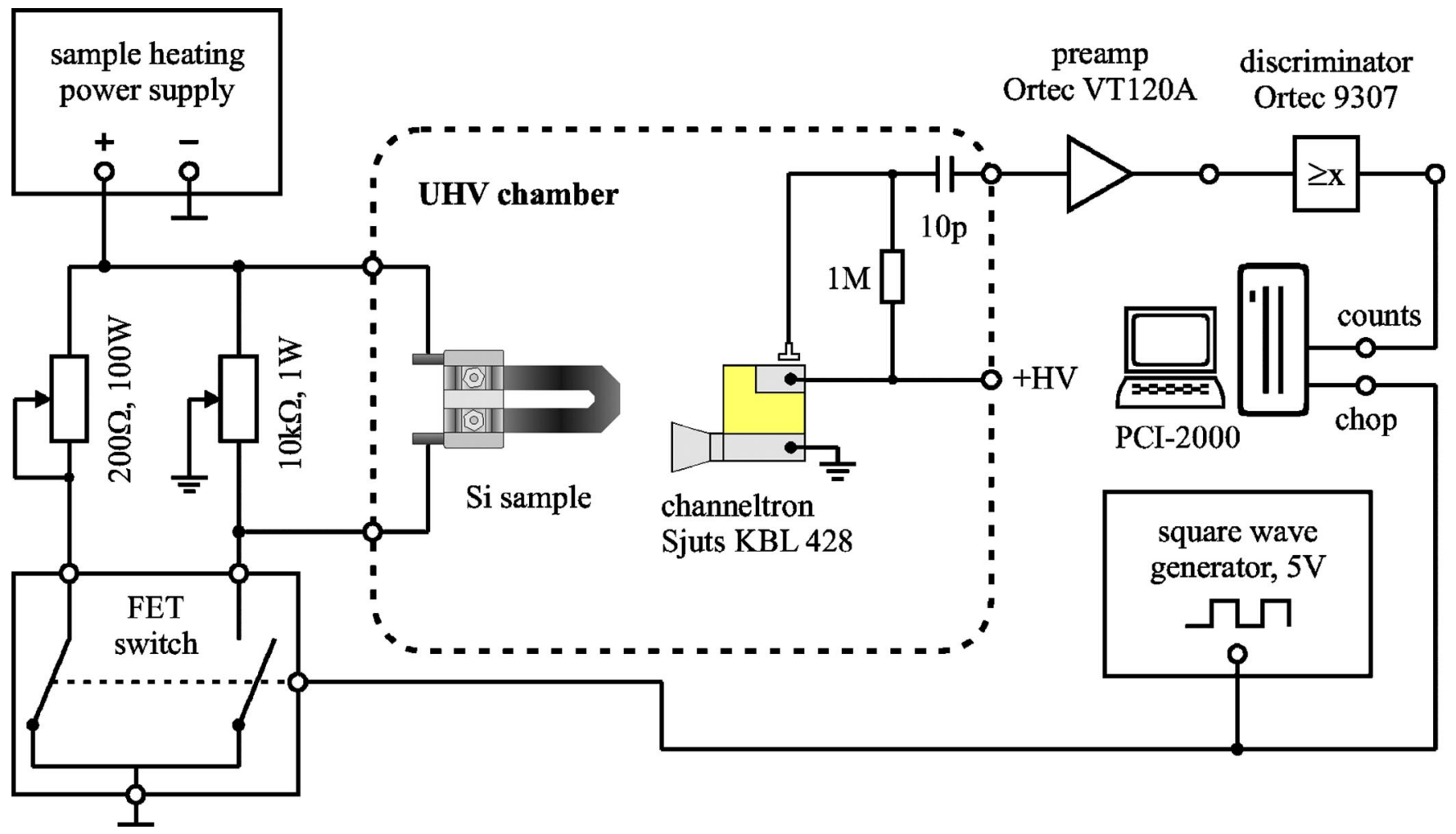

FIG. 2. (Color online) The principle of operation of the chopped sample heating: The sample heating power supply is connected and deconnected rapidly to the sample via a field effect transistor (FET) switch, which is controlled by a square wave signal. This signal also enables and disables the electron counting of the SPA-LEED data acquisition card and thus only electrons that saw a current-free sample are counted. 


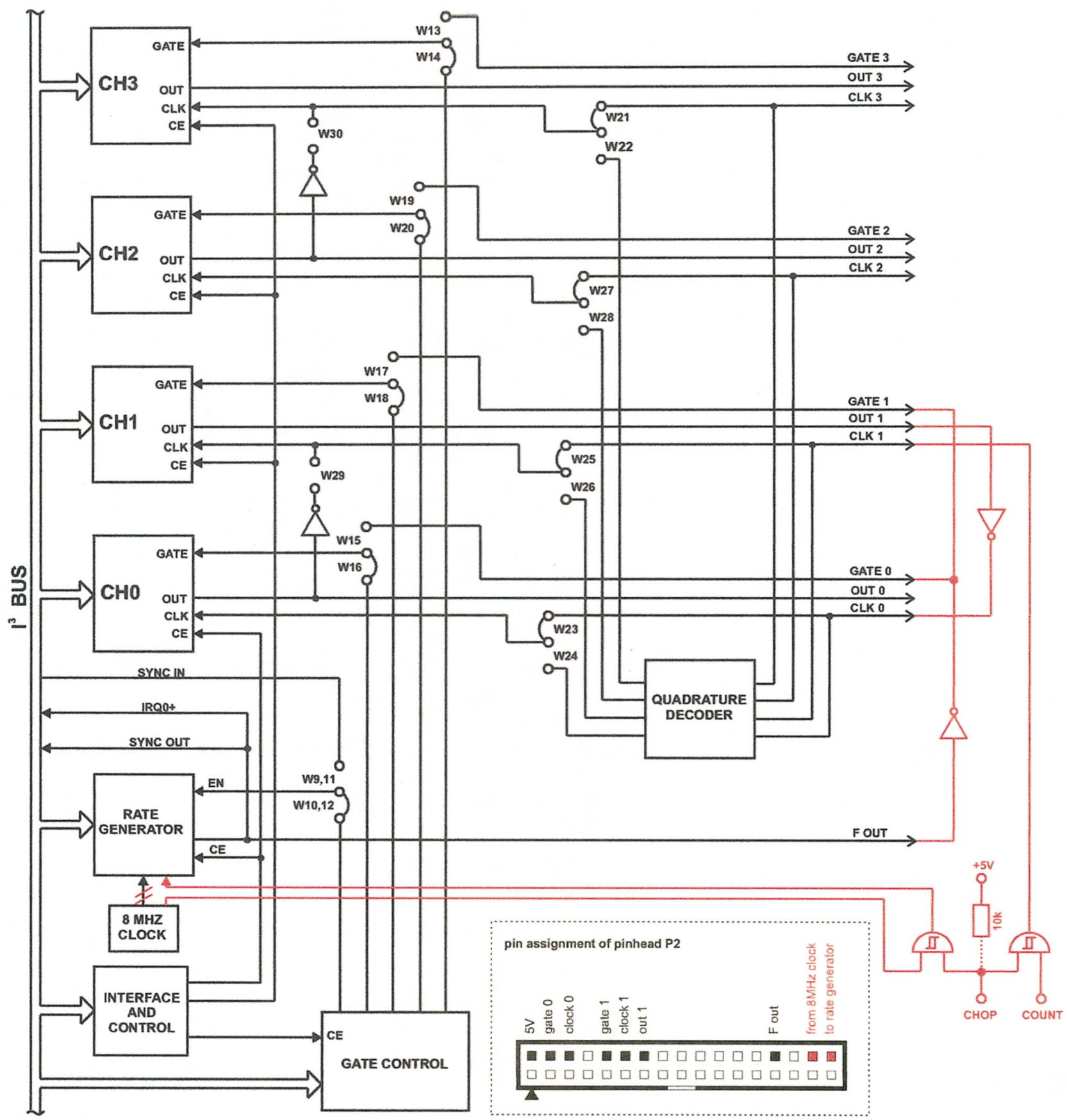

FIG. 3. (Color) Modification of the Intelligent Instrumentation PCI-20007M-1A timer module: The clock and the counter input are AND-connected to an external "chop" signal. The clock must be disconnected by removing a wire on the printed circuit board (PCB). If these signals to and from the $8 \mathrm{MHz}$ clock are connected to two reserved pins of pinhead P2, then all additional electronic devices can be placed on one small PCB, which can be directly plugged onto pinhead P2 of the timer module. The inverters are used to cascade the timers 0 and 1 . (Schematics taken from page 1.2 and 2.14 of the timer module's manual, reproduced with kind permission of Intelligent Instrumentation Inc.)

ronment, however, convection can become relevant. The loss of heat energy per time according to Newton's law of convection is given by

$$
\frac{d E}{d t}=\kappa \times A \times \Delta T,
$$

where $A$ is the surface of the sample, $\Delta T$ is the temperature difference between sample and the surrounding gas, and $\kappa$ is a constant describing the geometry. In the case of a flat rectangular sample $\kappa$ is about $15 \frac{\mathrm{W}}{\mathrm{m}^{2} \cdot \mathrm{K}}$ at normal pressure. Since
SPA-LEED is mainly used in UHV applications, convection will not be taken into account here.

The loss of heat energy $d E$ due to radiation of a thin sample with surface $A$ within a time period $d t$ and at a temperature $T$ is given by the Stefan-Boltzmann law

$$
\frac{d E}{d t}=2 \epsilon \sigma A T^{4},
$$

where $\epsilon$ is the emissivity of the sample in comparison to an ideal black body and $\sigma$ is the Stefan-Boltzmann-constant ${ }^{13}$ 


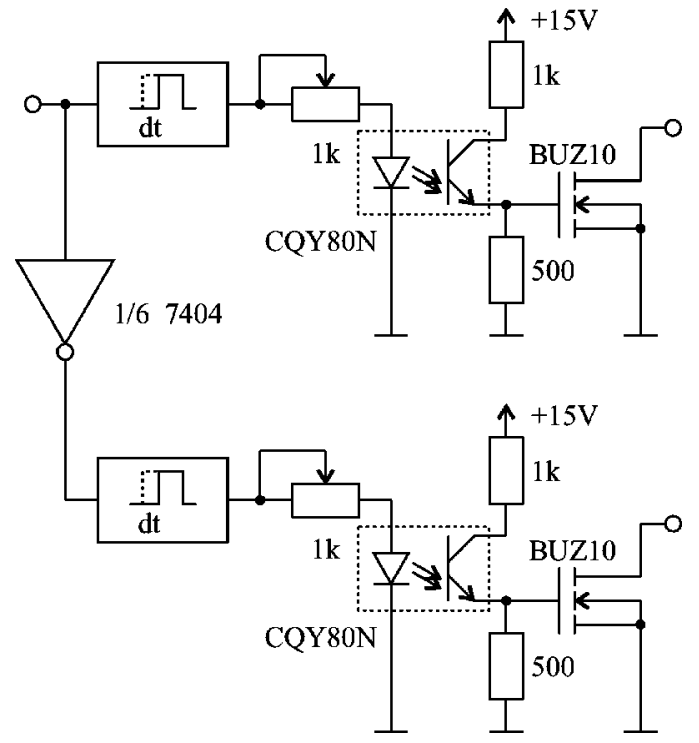

FIG. 4. Schematic of the fast switch. The FETs have to be mounted to a heatsink with a thermal resistance of less than $5 \mathrm{~K} / \mathrm{W}$. If a fast supply is used, only one half of this circuit may be needed (see Fig. 5). For details about the switch-on delays, see text.

$$
\sigma=\frac{\pi^{2}}{60} \frac{k_{B}^{4}}{\hbar^{3} c^{2}}=5.67051 \times 10^{-8} \frac{W}{m^{2} K^{4}} .
$$

The heat capacity $c^{14}$ is defined as the needed energy $d E$ for a change of temperature $d T$ per mass $m$ and this change $d T$ :

$$
c=\frac{1}{m} \frac{d E}{d T}=\frac{1}{\rho V} \frac{d E}{d T}
$$

where $V=A \times d$ is the volume of the sample and $\rho$ the density of the sample's material.

Solving Eqs. (1) and (2) for $d E$ and equating them produces the temporal rate of change of the sample's temperature

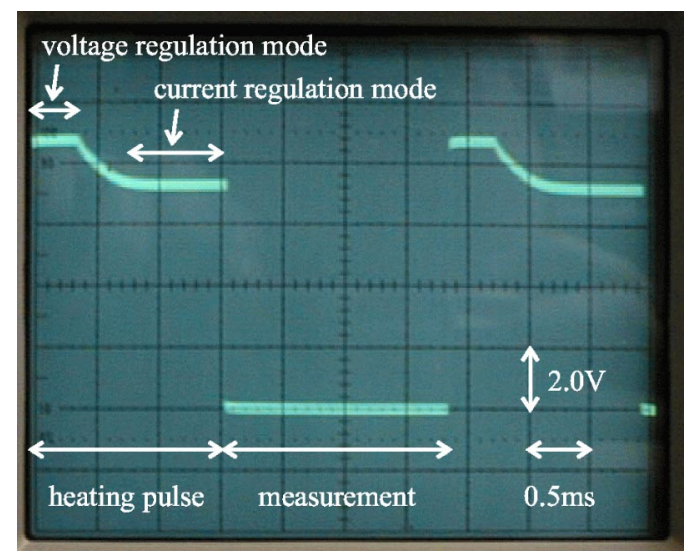

FIG. 5. (Color online) The voltage on the sample vs time shows the typical behavior of a fast sample heating power supply, if the second shunt resistor (see Fig. 2) is not used: The supply has to regulate the current between zero and a finite value, so it switches into a voltage regulation mode when the switch is closed. After about $0.4 \mathrm{~ms}$, a save current regulation mode is reached. In this case, the duty cycle is about $1: 1$, but if the alternative resistor is used, shorter heating pulses are useful.

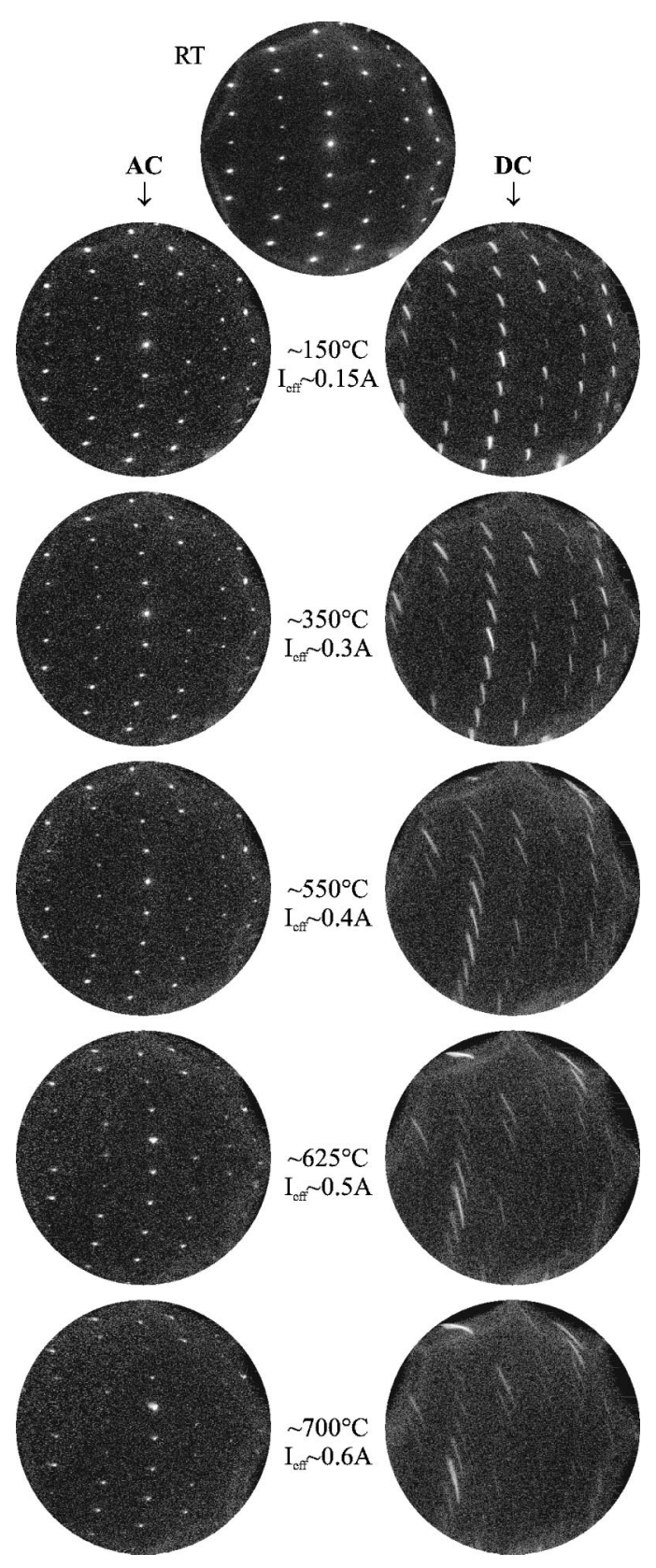

FIG. 6. Diffraction images at $E=160 \mathrm{eV}$ of a $(7 \times 7)$ reconstructed $\mathrm{Si}(111)$ sample (Ref. 28) in logarithmic scaling, acquired in the external geometry. Neither refocusing nor any changing of the ground point was done. Note the distorted diffraction spots and the movement of the octopole border (upper right) in the images taken with dc heating, and the decrease of the integral spot intensities due to thermal diffuse scattering in both series.

$$
\frac{d T}{d t}=\frac{2 \epsilon \sigma T^{4}}{d c \rho} .
$$

With the specific heat capacity $c_{\mathrm{Si}}=25 \frac{\mathrm{J}}{\mathrm{mol} \cdot \mathrm{K}}=880 \frac{\mathrm{J}}{\mathrm{kg} \cdot \mathrm{K}}$ (Ref. $15)$ and the mass density $\rho_{\mathrm{Si}}=2.33 \frac{\mathrm{g}}{\mathrm{cm}^{3}}$ (Ref. 16) of silicon, a sample's thickness of $d=0.1 \mathrm{~mm}$ and an emissivity of $\epsilon$ $=0.75$ (Refs. 17,18) one calculates for a typical temperature of measurement of $1100 \mathrm{~K}$ a cooling rate of the sample of

$$
\left(\frac{d T}{d t}\right)_{1100 \mathrm{~K}}=600 \frac{\mathrm{K}}{\mathrm{s}} .
$$

Thus, it is necessary to use heating pulse lengths of about 1 ms to keep the temperature of the sample constant within 
$1-2 \mathrm{~K}$. The power switch and the power supply must be fast enough to let the sample heating work in a save current regulation mode, which is necessary because of the decreasing resistance of silicon at higher temperatures. Also, the needed inverse chopper frequency is about the same as typically used counter gate times.

\section{B. Modification of the data acquisition hardware}

The modification of the data acquisition hardware is more complicated for a SPA-LEED than for conventional LEED systems ${ }^{19,20}$ since the chopping must be coordinated with the electron counting. If the synchronization between chopping and counting is not perfect, beats and moire patterns in the diffraction images are to be expected, because then the effective gate time of electron counting at each point in the reciprocal space scan depends on the phase shift between the "heat" signal and the scanning signal.

Thus, not only the counter input signal, but also its internal $8 \mathrm{MHz}$ master clock are AND-connected to an external enable signal (the inverted heater signal, see Fig. 3), and the complete operation of the data acquisition card is stopped during a heat pulse. Any modification of the SPA-LEED software is avoided.

The SPA-LEED is mostly used with the Intelligent Instrumentation $^{21}$ data acquisition card PCI-20001C-2A, equipped with a PCI-20006M-2 analog add-on module, a PCI-20007M-1A timer/counter module and a PCI-20021M1B 12 Bit analog output module. The timer/counter module has to be changed as shown in Fig. 3: A NAND gatter 74LS132 is used for the gating of the "counts" input and the gatetime via IC5, and two inverters of a 74LS04 are normally used to cascade the counters 0 and 1 of IC4. Even though most transistor-transistor logic (TTL) devices have internal pull-up resistors at their inputs (to reach a "high" state if the inputs are not connected), an additional pull-up resistor is used.

\section{Metal-oxide-semiconductor field-effect transistor switch}

The metal-oxide-semiconductor field-effect transistor (MOSFET) switch (see Fig. 4) is used to connect either the sample or an external resistor to the negative pin of the heating power supply. If that resistor is used and properly set to the same resistance as the sample, then the heating power supply works virtually in a dc mode. If the shunt is left away, this supply needs to be a fast one (see Fig. 5).

Most of the easy-to-use commercially available solid state relays are too slow for our application, because they contain electronic filters like Boucherot cells to suppress their switching noises in order to fulfill the requirements for electromagnetic emission. Thus, a small fast FET switch circuit as shown in Fig. 4 was designed: Two FETs are controlled via optocouplers, and a short switch-on delay of a few $10 \mathrm{~ns}$ is recommended to avoid the sample and the shunt resistor being both switched on in parallel. Such a delay is usually built with a monoflop and an AND gatter, ${ }^{23,24}$ but instead of the monoflop a series of slow standard TTL AND gatters is even better.
In our setup, a TTL circuit consisting of a quartz oscillator and subsequent read-set flipflop dividers is used as the square wave source, because it provides an extremely high frequency stability: The duty cycle ("on" to "off" ratio) must be absolutely constant in order to avoid temperature changes of the sample. To increase the effective measurement time, it is recommended to choose short heating pulse lengths (duty cycle $1 / 4$ to $1 / 10$ ).

\section{RESULTS}

As shown in Fig. 6, the chopped sample heating dramatically improves the diffraction images at higher sample currents: if the patterns are compared to the (field free) one at room temperature (RT), only an increase in the thermal diffuse scattering, ${ }^{25,26}$ described by the Debye-Waller factor $e^{-\left\langle\langle\vec{K} \cdot \vec{u}\rangle^{2}\right.} \propto e^{-T}$ and leading to a decrease of the integral spot intensities, is observed with nearly no deformations of the spots. $^{27}$

\section{ACKNOWLEDGMENTS}

The authors thank Kimberly and Kelly Roos for fruitful discussions and $\mathrm{J}$. Weiland of Intelligent Instrumentation Deutschland for the kind permission for using the original schematics. Financial support by the Deutsche Forschungsgemeinschaft is also gratefully acknowledged.

${ }^{1}$ C. Davisson and L. H. Germer, Phys. Rev. 30, 705 (1927).

${ }^{2}$ M. P. Seah and W. A. Dench, Surf. Interface Anal. 1, 2 (1979).

${ }^{3}$ K. D. Gronwald and M. Henzler, Surf. Sci. 117, 180 (1982).

${ }^{4}$ U. Scheithauer, G. Meyer, and M. Henzler, Surf. Sci. 178, 441 (1986).

${ }^{5}$ R. L. Park and H. E. Farnsworth, Rev. Sci. Instrum. 35, 1592 (1964).

${ }^{6}$ C. W. Caldwell, Rev. Sci. Instrum. 36, 1500 (1965).

${ }^{7}$ E. Bauer, Z. Metallkd. 63, 437 (1972).

${ }^{8}$ M. G. Lagally and J. A. Martin, Rev. Sci. Instrum. 54, 1273 (1983).

${ }^{9}$ M. Horn-von Hoegen, Z. Kristallogr. 214, 1 (1999).

${ }^{10}$ P. Zahl and M. Horn-von Hoegen, Rev. Sci. Instrum. 73, 2958 (2002).

${ }^{11}$ A. J. Schell-Sorokin and R. M. Tromp, Phys. Rev. Lett. 64, 1039 (1990).

${ }^{12}$ P. Zahl, P. Kury, and M. Horn-von Hoegen, Appl. Phys. A: Mater. Sci. Process. 69, 481 (1999).

${ }^{13}$ P. A. Tipler, Physik (Spektrum akademischer, Heidelberg, 1995).

${ }^{14}$ In this case, the heat capacity at constant pressure $c_{P}$ is used.

${ }^{15}$ V. M. Glazov, A. S. Paschkin, and M. S. Mikhailova, Scand. J. Metall. 30, 388 (2001).

${ }^{16} \mathrm{http}: / /$ www.webelements.com

${ }^{17} \mathrm{R}$. Hild, $\mathrm{PhD}$ thesis (unpublished).

${ }^{18}$ Here, the emissivity $\epsilon$ describes the radiation of the sample in comparison to a black body, but averaged over all wavelengths $\epsilon(T)=\langle\epsilon(\lambda, T)\rangle_{\lambda}$, so that the Stefan-Boltzmann law is fulfilled.

${ }^{19}$ J. C. Tracy, Rev. Sci. Instrum. 39, 1300 (1968).

${ }^{20}$ G. Held, S. Uremovic, C. Stellwag, and D. Menzel, Rev. Sci. Instrum. 67, 378 (1996).

${ }^{21}$ http://www.instrument.com

${ }^{22}$ see http://www.sjuts.com

${ }^{23}$ U. Tietze and C. Schenk, Halbleiter-Schaltungstechnik, 12th ed. (Springer, Berlin, 2002).

${ }^{24}$ J. Kammerer, W. Oberthür, J. Piegsa, and H. J. Siedler, Elektronik III (Grundschaltungen) (Pflaum, Munich, 1980).

${ }^{25}$ J. Aldag and R. M. Stern, Phys. Rev. Lett. 14, 857 (1965).

${ }^{26}$ A. U. MacRae and L. H. Germer, Phys. Rev. Lett. 8, 489 (1962).

${ }^{27}$ However, it should be noted that normally the strong distortions in the diffraction images taken with dc heating can either be reduced by refocusing the electron gun and/or changing the position of the ground potential point on the sample, or only a one-dimensional profile may be needed: then the diffraction spots can be elongated in one direction without compromising the measurement.

${ }^{28}$ K. Takayanagi, Y. Tanishiro, S. Takahashi, and M. Takahashi, Surf. Sci. 164, 367 (1985). 